\title{
Composition and strain dependence of band offsets at metamorphic $\operatorname{In}_{x} \mathrm{Ga}_{1-x} \mathrm{As} / \mathrm{In}_{y} \mathrm{Al}_{1-y} \mathrm{As}$ heterostructures
}

\author{
A. Stroppa ${ }^{1,2, *}$ and M. Peressi ${ }^{1,2, H}$ \\ ${ }^{1}$ Dipartimento di Fisica Teorica, Università di Trieste, \\ Strada Costiera 11, I-34014 Trieste, Italy \\ ${ }^{2}$ INFM DEMOCRITOS National Simulation Center, Trieste, Italy
}

(Dated: September 24, 2018)

\begin{abstract}
We have studied the $\operatorname{In}_{x} \mathrm{Ga}_{1-x} \mathrm{As} / \mathrm{In}_{y} \mathrm{Al}_{1-y} \mathrm{As}$ (001) interface using first-principles ab-initio pseudopotential calculations, focusing on the effects of alloy composition and strain state on the electronic properties. In particular we estimate a valence band offset (VBO) of $0.11 \mathrm{eV}$ (InGaAs higher), including spin-orbit and self-energy corrections, for a strain-compensated configuration with homogenous composition $x=y=0.75$ on a lattice-matched substrate. Unintentional composition fluctuations which are typically limited to a few percent and different short-range order effects give rise only to small variations on the $\mathrm{VBO}$, of the order of $0.1 \mathrm{eV}$ or less, whereas intentional substantial changes in the alloys composition allow to achieve a high tunability of band offsets. We predict a VBO varying in a range of about $1.1 \mathrm{eV}$ for interfaces between the pure arsenides in different strain states as extreme cases of composition variation at $\operatorname{In}_{x} \mathrm{Ga}_{1-x} \mathrm{As}_{1} / \mathrm{In}_{y} \mathrm{Al}_{1-y} \mathrm{As}$ heterostructures.

PACS numbers: PACS: 73; 73.40.Kp; 73.20.-r; 68.35.-p
\end{abstract}




\section{INTRODUCTION}

GaAs, AlAs, and InAs form the family of common-anion III-V conventional semiconductors covering the widest possible range of energy gaps, apart from nitrides. 1 They are therefore particularly suitable to be combined into alloys to form $\operatorname{In}_{x} \mathrm{Ga}_{1-x} \mathrm{As} / \mathrm{In}_{y} \mathrm{Al}_{1-y} \mathrm{As}$ heterojunctions whose electronic properties can be easily tailored according to the technological needs, acting on composition to control and intentionally modify the valence and conduction band offsets (VBO and CBO), namely for band-offset engineering. 2.3.4.5

The use of alloys in heterojunctions has also some drawbacks. Beside controlled variations in the average composition, unintentional composition inhomogeneities could be present in epitaxially grown alloys and heterostructures, as detected by tunneling microscope techniques. ${ }^{6.7 .8}$ Their origin can be ascribed to several mechanisms: inhomogeneous incorporation of the alloy components during growth, atomic diffusion at the surface during growth induced by strain inhomogeneities arising from stress relaxation and/or interface roughening, and also post-growth atomic interdiffusion with or without thermal annealing. Although in high-quality alloy-based nanostructures and devices such inhomogeneities are minimized, their residual occurrence can affect the electronic and optical properties. ${ }^{6.9 .10 .11 .12}$

Another source of variations of the electronic properties in alloy-based heterojunctions is the occurrence of spontaneous ordering in the constituting alloys. In the last years considerable theoretical and experimental effort have been devoted in investigating the effect on the band alignments and related properties. ${ }^{13.14 .15 .16}$

It is important for device design not only to predict the value of band offsets at heterojunctions with given composition, but also to estimate the effects of both composition fluctuations and ordering. We address this problem here, focusing on the $\operatorname{In}_{x} \mathrm{Ga}_{1-x} \mathrm{As}_{\mathrm{s}} / \mathrm{In}_{y} \mathrm{Al}_{1-y} \mathrm{As}$ heterojunction and studying by accurate ab-initio simulations: $(i)$ the band offsets at a given nominal composition $x=y=0.75$, presently of particular technological interest, ${ }^{17}$ considering different structural configurations; $(i i)$ the effect of possible realistic composition fluctuations with respect to the nominal one.

A computational approach that has been used to simulate several alloy-based heterojunctions, such as $\mathrm{AlGaP} / \mathrm{GaP}^{18}$, GaAlAs/GaAs${ }^{19}$ and AlGaInAs-based heterostructures ${ }^{20}$, is the virtual crystal approximation (VCA). This approach considers an average crystalline field acting on the electrons rather than the effect of the individual atoms and therefore is 
particularly valid where the elements forming the alloy are very similar, such as the Al and Ga atoms in the examples above. By its nature, the VCA has intrinsic limitations in the atomic-scale description of the materials (e.g. it cannot describe lattice distortions). Furthermore, it has to be applied with care also in the calculation of macroscopic properties. It is known for instance that it gives positive deviations from the experimentally observed linear behaviour of the alloy lattice parameter with composition (Vegard's law) which is instead recovered using a fully atomistic approach, with "real" instead of "virtual" atoms. To overcome this limitation, the VCA could be applied to calculate the average electronic properties only after having considered the correct structure ${ }^{2,21}$ In a previous work we have used the VCA for a preliminar study of the electronic properties of $\operatorname{In}_{x} \mathrm{Ga}_{1-x} \mathrm{As}_{\mathrm{s}} / \mathrm{In}_{y} \mathrm{Al}_{1-y} \mathrm{As}$ heterojunctions. ${ }^{2}$ Here we study more accurately the case $x=y=0.75$ using a fully atomistic approach with "real" atoms. This is mandatory in order to investigate the effects of realistic composition fluctuations and ordering. ${ }^{22}$

We predict a VBO of $0.11 \mathrm{eV}$ (InGaAs higher) and we estimate that realistic composition fluctuations and ordering effects are small and not exceeding $\approx 0.1 \mathrm{eV}$. For completeness, we have also estimated the maximum range of tunability of the offsets at $\operatorname{In}_{x} \mathrm{Ga}_{1-x} \mathrm{As} / \operatorname{In}_{y} \mathrm{Al}_{1-y} \mathrm{As}$ heterojunctions by varying intentionally the composition of the alloys with variations of $x$ and $y$ separately up to the limit of pure binary semiconductors.

In the next Section we present our computational approach. Section III is devoted to the preliminar study of the three binaries (GaAs, AlAs and InAs) constituting the junctions, both in their bulk phase and in strained configurations, and of the two $\operatorname{In}_{0.75} \mathrm{Ga}_{0.25} \mathrm{As}$ and $\mathrm{In}_{0.75} \mathrm{Al}_{0.25} \mathrm{As}$ alloys in strain-free configurations. In Sect. IV we report the results for the $\operatorname{In}_{0.75} \mathrm{Ga}_{0.25} \mathrm{As} / \mathrm{In}_{0.75} \mathrm{Al}_{0.25}$ As heterojunction in the hypothesis of a uniform homogeneous composition and we discuss the effects of different ordering and composition fluctuations. We complete the work considering in Sect. $\mathrm{V}$ the interfaces between the binaries, in different possible strain configurations. Finally, we draw our conclusions.

\section{COMPUTATIONAL DETAILS}

We perform state-of-the-art first-principles non-relativistic pseudopotential self-consistent calculations within the Local Density Approximation (LDA) to Density Functional Theory (DFT) using the PWSCF code. ${ }^{23}$ We address the reader to Appendix A for details con- 
cerning the inclusion of spin-orbit corrections a posteriori. Periodically repeated supercells are used to simulate both the bulk alloys and the heterojunctions. We use norm-conserving pseudopotentials with $d$ electrons of $\mathrm{Ga}$ and In in the core and the non-linear core correction for In. This choice is very convenient since-compared with a complete neglect of the effects of $d$ electrons-with a very limited computational effort it improves significantly the description of the structural and electronic parameters, as it is widely reported in the literature for the traditional III-V compounds. We address the reader to Appendix B for a deeper discussion based on additional accurate all-electron calculations on the combined effects of $p-d$ coupling and spin-orbit and/or strain splitting in these systems.

The plane wave basis set is expanded up to a kinetic energy cutoff $E_{\text {cut }}=20$ Ry which gives well converged equilibrium structural and electronic properties for the systems considered. Test with 25 Ry of cutoff have been done for the binary and ternary compounds. The integration over the Brillouin zone is performed using the $(4,4,4)$ Monkhorst-Pack mesh for the FCC cell and corresponding meshes for the various supercells.

For all the heterojunctions we consider pseudomorphic growth conditions, i.e. we fix the in-plane lattice-constant $a_{\|}$and the possible lattice mismatch between the constituting materials is accommodated without defects by a strain field $\epsilon$. Far from the interface the strain field is uniform and can be described by the perpendicular lattice parameter $a_{\perp}$. The macroscopic theory of elasticity predicts: $:^{24.25}$

$$
\begin{aligned}
a_{\perp} & =a_{0}\left[1-2\left(\frac{c_{12}}{c_{11}}\right) \epsilon_{\|}\right], \\
\epsilon_{\|} & =\frac{a_{\|}}{a_{0}}-1, \quad \epsilon_{\perp}=\frac{a_{\perp}}{a_{0}}-1,
\end{aligned}
$$

where $a_{0}$ is the cubic lattice parameter of the strained material, $c_{11}$ and $c_{12}$ are its elastic constants, and $\epsilon_{\|}$and $\epsilon_{\perp}$ are the components of the strain in direction parallel and perpendicular with respect to the substrate. The use of Eq. (1) with $c_{i j}$ calculated by first principles to determine $a_{\perp}$ is equivalent to a direct optimization of the atomic positions along the growth direction by stress and total energy minimization, but it is more convenient since a change of the substrate does not require a new self-consistent calculation. For the heterojunctions we take the prediction of Eq. (1) as a starting guess and then we perform a further optimization of the atomic position in order to obtain the correct interatomic distances also in the interface region, where they can differ ${ }^{26}$ We optimize the internal supercell structure until forces are less than $0.02 \mathrm{eV} / \AA$. The effects of these final structural optimizations on 
VBO amount up to $\approx 0.1 \mathrm{eV}$.

As explained in details in Ref. 2, we calculate the VBO by summing two contributions: $V B O=\Delta E_{v}+\Delta V$, i.e. the band structure term $\Delta E_{v}$ (the energy difference between the relevant top band-edges of the two materials in their appropriate strain state, measured with reference to the average electrostatic potential in the corresponding bulk crystal), and the electrostatic potential lineup $\Delta V$ containing all interface-specific effects and extracted from the self-consistent charge distribution in the heterojunction supercells. We report a positive value for $\mathrm{VBO}(\mathrm{CBO})$ at the $\mathrm{A} / \mathrm{B}$ interface if the valence band top edge (conduction band bottom edge) in $\mathrm{A}$ is higher than in $\mathrm{B}$. Our final estimates of $\mathrm{VBO}(\mathrm{CBO})$ include also spin orbit effects added a posteriori using experimental data (see Appendix A).

\section{BULK CONSTITUENTS}

\section{A. Binary semiconductor compounds}

Table 【 summarizes the calculated relevant equilibrium structural and electronic properties of the bulk binary compounds. The agreement between the experimental and the calculated lattice constants is within $\approx 1 \%$ which is acceptable since the mismatch between InAs and GaAs (AlAs) is much larger $(\approx 7 \%$, see Table $c_{11}, c_{12}$ and bulk moduli are equal to the experimental values within a few $\%$ for GaAs and AlAs, whereas the discrepancy is larger (by approximately 10-20 \%) for InAs. Incidentally we note that the value of bulk moduli calculated directly via the Murnaghan 27 equation of

state from the total energy curve satisfies the well known relation: $\mathrm{B}=\frac{c_{11}+2 c_{12}}{3}$ within the numerical accuracy (estimated to be $\approx 10 \mathrm{Kbar}$ ). Therefore, our choice of pseudopotentials is justified and we can afford the study of the alloys and of their heterojunctions on a realistic ground, with the possibility of a correct description of internal distortions.

The energy gaps are systematically smaller than the experimental values, as typically occurs in DFT-LDA, the worst case being AlAs.

\section{B. Ternary semiconductor alloys: $\operatorname{In}_{0.75} \mathbf{G a}_{0.25} \mathrm{As}$ and $\operatorname{In}_{0.75} \mathrm{Al}_{0.25} \mathrm{As}$}

We study here the bulk properties of the pseudobinary semiconducting alloys which are constituting the heterostructure at the nominal composition $x=y=0.75$. For definiteness 
we focus first on the $\operatorname{In}_{0.75} \mathrm{Ga}_{0.25}$ As alloy.

We consider only three different very simple ordered structures corresponding to the most homogeneous distributions of the different cation types (i.e. on the smallest possible scale compatible with the composition): Luzonite (labelled "L", with 8 atoms unit-cell), Famatinite ("F", with 16-atom unit-cell) and (001) 1+3 superlattice ("S", with 8-atom unitcell). In the first one the Ga atoms are arranged in a SC lattice; therefore this structure has the same cubic symmetry which is typical of the pure binary semiconductors. ${ }^{28}$ In the second structure the Ga atoms are arranged in a tetragonal body-centered structure, and their relative distance is greater than or at least equal to one cubic lattice parameter. The third structure is anisotropic, and it is the only one (among the three considered) where Ga atoms are next nearest neighbors. Larger supercells or alternative approaches accounting for compositional disorder on larger scales would be necessary for a complete treatment of the possible effects of randomness and for statistical analysis, ${ }^{29.30 .31}$ but this goes beyond the purpose of the present work.

Although the structures considered here are very simple, they allow us to catch the main structural features of the alloy, that we summarize in the following.

The calculated equilibrium average lattice parameter is $a_{\text {alloy }}=5.86 \AA$ for all the three structures, almost equal to the Vegard's value.

In each structure, the nearest-neighbor (NN) pairs are of two types (Ga-As and In-As), with an occurrence proportional to the corresponding cationic concentration; the calculated individual NN distances (see Table III) show a bimodal distribution centered around two values quite close to the bulk-like Ga-As $(2.40 \AA)$ and In-As $(2.58 \AA)$ values, as typically observed in most pseudobinary semiconductor alloys: ${ }^{32}$ more precisely, the Ga-As distances in the alloys are within the range 2.41-2.45 $\AA$, whereas the In-As distances are within 2.56$2.60 \AA$. The weighted average of all the anion-cation bond lengths is $2.54 \AA$, coinciding with the NN distance in the ideal unrelaxed structure.

The pattern of the next nearest-neighbor (NNN) environment is more complex, due to the presence of both As-As and cation-cation pairs. The anionic sublattice is rather distorted with respect to the ideal zincblende structure, due to different types of NN cations surrounding As, whereas the cationic sublattice is only slightly distorted, having only NN As atoms. As a consequence, the NNN distances of the cation-cation pairs (In-In, Ga-In and $\mathrm{Ga}-\mathrm{Ga})^{33}$ are quite close to the corresponding common value in the ideal undistorted 
structure, i.e. $4.14 \AA$ : they are exactly equal to this value in the $L$ and $F$ structures, and are within a range of $0.15 \AA$ (from 4.07-4.22 $\AA$ ) in the $S$ structure. The As-As NNN distances are rather scattered with respect to the ideal undistorted value, even in the $L$ structure where the high symmetry limits the internal distortions: the As-As NNN distances have a quite broadened distribution in a range of about $0.44 \AA$, from $3.92 \AA$ to $4.36 \AA$.

The $\mathrm{In}_{0.75} \mathrm{Al}_{0.25}$ As alloy shows structural properties very similar to the one with $\mathrm{Ga}$, as it can be seen again from Table III. Neglecting the tiny mismatch between GaAs and AlAs, it is perfectly lattice-matched with $\mathrm{In}_{0.75} \mathrm{Ga}_{0.25} \mathrm{As}$.

The electronic structures of the alloys in the three different structures are rather similar, as shown by the comparison of their band structures and of their density of states in Figs. 1 and 2, with some differences. In all the three different structures considered, the calculated gaps are slightly smaller than the linear interpolation between those of the parent endpoints, suggesting a small positive bowing in qualitative agreement with the experimental data available: $\frac{1}{-}$ within $\mathrm{LDA}$, we calculate $E_{g}$ in the range $0.49-0.55 \mathrm{eV}$ for $\operatorname{In}_{0.75} \mathrm{Ga}_{0.25} \mathrm{As}$ and in the range $0.82-0.90 \mathrm{eV}$ for $\mathrm{In}_{0.75} \mathrm{Al}_{0.25} \mathrm{As}$ (which has a direct minimum gap at this composition), to be compared with the linear interpolations $0.65 \mathrm{eV}$ and $0.90 \mathrm{eV}$ respectively.

Although we do not perform an exhaustive study, we can however give a rough estimate of the effect of the structural order on the energy gap from the variations of the calculated values for the different ordered structures considered here: the maximum calculated variation of the gap is $0.08 \mathrm{eV}$, small but higher than our relative numerical accuracy which amounts to 20-30 meV. Incidentally, we notice that a comparable effect $(0.06 \mathrm{eV})$ has been determined from photoluminescence excitation spectroscopy as band gap difference between the ordered and disordered epitaxial $\mathrm{Ga}_{x} \operatorname{In}_{1-x} \mathrm{P}{ }^{15}$ Higher band-gap reductions (up to $0.25 \mathrm{eV}$ and 0.18 $\mathrm{eV}$ for $\mathrm{In}_{0.5} \mathrm{Ga}_{0.5} \mathrm{As}$ and $\mathrm{In}_{0.5} \mathrm{Al}_{0.5} \mathrm{As}$ respectively) are instead predicted theoretically for a fully disordered $\rightarrow \mathrm{CuPt}$-[111]-ordered transition, which is accompanied by the formation of important piezoelectric fields: ${ }^{34}$ however, in real samples the degree of spontaneous order is not perfect, and the effect is expected to be $\leq 0.1 \mathrm{eV}$. We also notice that a band gap reduction up to $0.06 \mathrm{eV}$ is predicted in Ref. 12 in epitaxial $\operatorname{In}_{0.5} \mathrm{Ga}_{0.5}$ As alloy, due to lateral composition modulation (which can be also seen as a ordering effect, but on a different length scale) with respect to an homogeneous configuration. 


\section{THE $I n_{0.75} G a_{0.25} A s / I n_{0.75} A l_{0.25} A s$ HETEROJUNCTION}

We consider the heterojunction between the two alloys $\operatorname{In}_{0.75} \mathrm{Ga}_{0.25}$ As and $\operatorname{In}_{0.75} \mathrm{Al}_{0.25} \mathrm{As}$ on a lattice-matched (001) substrate. This heterostructure indeed has been successfully grown in pseudomorphic and almost unstrained configuration by inserting InAlAs buffers with graded In composition on GaAs substrates. ${ }^{17.35 .36 .37 .38}$ Very recently it has been reported on the achievement of a two-dimensional electron gas in these quantum well structures, with reduced carrier density and high mobility 17

We recall here that also other particular compositions are of technological interest. $\operatorname{In}_{x} \mathrm{Ga}_{1-x} \mathrm{As} / \mathrm{In}_{y} \mathrm{Al}_{1-y} \mathrm{As}$ heterojunctions grown with an In concentration $x \approx 0.3$ on an unstrained metamorphic buffer are widely used in microwave and optoelectronic devices. ${ }^{39.40}$ Devices based on $\operatorname{In}_{0.53} \mathrm{Ga}_{0.47} \mathrm{As}$ and $\mathrm{In}_{0.52} \mathrm{Al}_{0.48} \mathrm{As}$ alloys are also developed for a wide variety of optoelectronic and high-speed electronic applications, since for these particular compositions they can be easily grown lattice-matched to the commercial InP substrate.

We simulate the $\operatorname{In}_{0.75} \mathrm{Ga}_{0.25} \mathrm{As} / \mathrm{In}_{0.75} \mathrm{Al}_{0.25} \mathrm{As}$ interface by using tetragonal supercells made of three slab unit cells for each constituent alloy along the (001) direction. For the sake of definiteness we consider the same structure and configuration for the two alloys which form the interface.

Since the alloys in the $L$ and $F$ structures are very similar in terms of structural and electronic properties, we consider for the calculation of the VBO only the $L$ and the $S$ structures. For the $L$ structure the heterojunction supercell contains six simple cubic cells for a total length normal to the interface of $6 a_{0}=35.16 \AA$, with $a_{0}$ the average lattice parameter of the alloys. The cation profile along (001) is: ...X-In-X-In-Y-In-Y-In-.., where $X$ indicates the mixed-cation planes with $50 \% \mathrm{Ga}$ and $50 \% \mathrm{In}$, and $Y$ refers to the mixedcation planes with $50 \% \mathrm{Al}$ and $50 \%$ In. We report in Fig. 3. upper panels, the macroscopic averages $^{2}$ of charge (solid lines) and potential (dashed lines). The calculated VBO with the optimized atomic positions and with the spin-orbit effects included a posteriori ${ }^{2.41}$ is 0.07 $\mathrm{eV}$, with a numerical error of the order of 20-30 meV. Self-energy corrections to the valence band top edges have to be considered for a more realistic estimate. From the values given in Ref. 42, properly scaled to be adapted to our calculations, ${ }^{43}$ the self-energy correction to our LDA VBO is $0.04 \mathrm{eV}$, thus giving a final $\mathrm{VBO}=0.11 \mathrm{eV}$.

The VBO obtained from supercell calculations can be compared with the linear inter- 
polation between the parent end-points: the GaAs/AlAs interface $(x=y=0)$ and the trivial case of the InAs/InAs homojunction $(x=y=1)$. The calculated LDA VBO at the unstrained GaAs/AlAs interface, including spin-orbit effects but not self-energy corrections, is $\mathrm{VBO}=0.44 \mathrm{eV}$, which well compares with previously reported values. $\stackrel{2}{2}^{2}$ The linear interpolation for $x=y=0.75$ gives $0.11 \mathrm{eV}$, to be compared with the supercell calculation giving $0.07 \mathrm{eV}$. We thus confirm in this work - in sign and magnitude within the numerical accuracy - the deviation from linearity that was roughly estimated in Ref. 2 with slightly different technical details and composition.

In order to estimate the possible effects of short-range order/disorder, we then consider the case where both alloys are simulated with the $S$ structure, which is a limiting case of maximum ordering in a particular direction, here considered as the growth direction. In this case the total length of the supercell normal to the interface is equal to $12 a_{0}=70.32 \AA$. The cation profile is the following: ... In $-G a-I n-I n-I n-G a-I n-I n-I n-A l-I n-\ldots$ The charge and potential profiles are shown in the lower part of Fig. 3. Remarkably, also in this case the calculated VBO is $0.07 \mathrm{eV}$, although differently divided between $\Delta E_{v}$ and $\Delta V$. ¿From our study, however, we cannot fully exclude an effect of ordering on the VBO: we can only conclude that this effect, if present, is small and could be hidden by our numerical accuracy.

A further comment is in order concerning the effect on $\mathrm{VBO}$ of the possible composition fluctuations: we account for them considering that the real composition in the region where the band offsets are detected could be sligthly different from the nominal one. In high-quality samples this difference should not exceed $\approx \pm 5 \%$, so that the corresponding variation in the VBO should be of the order of our numerical accuracy, less than $0.05 \mathrm{eV}$, estimated from the VBO between the end-points.

Finally, we can estimate the CBO for the heterojunction from the calculated VBO and from the experimental data for the gaps of the alloys, $\frac{1}{2}$ taking into account a quadratic interpolation between the end-points: we obtain $\mathrm{CBO}=-0.29 \mathrm{eV}$, with $\mathrm{In}_{0.75} \mathrm{Al}_{0.25} \mathrm{As}$ higher. The CBO is more sensitive than the VBO to the effects of order: although we do not find appreciable variations for the $\mathrm{VBO}$, variations up to about $0.14 \mathrm{eV}$ in the $\mathrm{CBO}$ could be predicted simply by combining the effects of the variations of the band gap in the two alloys discussed in the previous Section. 


\section{HETEROJUNCTIONS BETWEEN THE (GAAS, ALAS, INAS) BINARIES}

We focus now on the three limiting cases of the $\operatorname{In}_{x} \mathrm{Ga}_{1-x} \mathrm{As} / \mathrm{In}_{y} \mathrm{Al}_{1-y}$ As heterojunction corresponding to interfaces between pure binaries: (a) $(x, y)=(0,0),(\mathrm{b})(x, y)=(1,0),(\mathrm{c})$ $(x, y)=(0,1)$, i.e. GaAs/AlAs, InAs/AlAs and GaAs/InAs interfaces respectively. In these simple cases the supercells contain 12 atoms and have a total length equal to approximately $3 a_{0}$, depending on the substrate and consequently on the strain state. We are going to consider different possible strain configurations within the range of pure GaAs (AlAs) or pure InAs substrate. Although the accidental local formation of islands of pure GaAs and AlAs binaries at $\operatorname{In}_{x} \mathrm{Ga}_{1-x} \mathrm{As} / \mathrm{In}_{y} \mathrm{Al}_{1-y}$ As heterojunction is rather unrealistic for the In-rich nominal composition that we have considered, it could be eventually possible for InAs. More realistically, the cases that we are going to discuss correspond to different heterostructures intentionally grown. Since the purpose of this Section is to estimate the possible range of variability of the offsets, for the sake of semplicity we will report VBO and CBO values without self-energy corrections: this choice does not change our conclusions.

\section{A. Alloy substrate}

We first consider the case of in-plane lattice constant between those of GaAs (AlAs) and InAs. For the sake of definiteness we consider the one of the $\operatorname{In}_{0.75} \mathrm{Ga}_{0.25} \mathrm{As}$ and $\operatorname{In}_{0.75} \mathrm{Al}_{0.25} \mathrm{As}$ alloys, $a_{\|}=5.86 \AA$.

Each pure binary semiconductor in this condition is strained: GaAs and AlAs are under a tensile in-plane strain $\left(\epsilon_{\|}=0.058\right.$ and 0.047 respectively) and therefore shrink along the growth direction, whereas InAs is under a compressive in-plane strain $\left(\epsilon_{\|}=-0.016\right)$ and therefore elongates along the growth direction. Considering the theoretical lattice parameters and elastic constants in Table 凹 we predict $a_{\perp}=5.28,5.34,6.07 \AA$ and $\epsilon_{\perp}=-0.048$, -0.047, 0.018 for GaAs, AlAs and InAs respectively. A full optimization of the atomic positions gives a small overstrain in the interface region.

From the supercells with optimized atomic positions we calculate the potential lineups, on top of which we add the band edges of the binaries in their appropriate strain state (see Table IV and Appendix A 1). Finally, the VBO including macroscopic and local strain and spin-orbit effects (not self-energy corrections) is: $0.16 \mathrm{eV}, 0.30 \mathrm{eV}, 0.44 \mathrm{eV}$ for the 
GaAs/InAs, InAs/AlAs, and GaAs/AlAs strained interfaces (see Fig. [. panel c)).

The conduction band bottom edge is also affected by the strain field. Details of the calculations are given in Appendix A2, distinguishing the cases of direct/indirect gap. Using together our numerical calculations and experimental data, the best estimate for the gaps of the three binaries strained on the in-plane alloy lattice constant are: $E_{g, G a A s}^{\text {strained }}=0.69$ $\mathrm{eV}, E_{g, I n A s}^{\text {strained }}=0.44 \mathrm{eV}$ and $E_{g, A l A s}^{\text {strained }}=1.76 \mathrm{eV}$ (indirect). ¿From the previously calculated VBO we can therefore easily predict also the $\mathrm{CBO}: 0.41 \mathrm{eV},-1.02 \mathrm{eV}$, and $-0.63 \mathrm{eV}$ for GaAs/InAs, InAs/AlAs, and GaAs/AlAs heterojunctions respectively. We summarize such results again in Fig. 固, panel c).

A different kind of band alignment is predicted for the GaAs/AlAs and AlAs/InAs case with respect to GaAs/InAs. Due to the large gap of AlAs, in the former cases the conduction and valence band edges of the smaller bandgap material (GaAs or InAs) are both within the bandgap of AlAs, that is a type I alignment. At variance, in the latter case, we have a type II alignment, with an effective bandgap of the heterostructure of $0.28 \mathrm{eV}$.

Finally, we notice that the predicted VBO and CBO for the three interfaces well satisfy the transitivity rule within the numerical error, confirming the validity of the linear response theory ${ }^{2}$ for the class of isovalent heterojunctions.

\section{B. Pure binary substrates}

We have studied also the VBO and the $\mathrm{CBO}$ for same three interfaces between the binaries, but considering $a_{\|}=a_{0}(\operatorname{InAs})=5.96 \AA$, (Fig. 团 panel b)), and $a_{\|}=5.57 \AA \approx$ $a_{0}(\mathrm{GaAs}) \approx a_{0}(\mathrm{AlAs})$ (Fig. [4 panel d)), corresponding respectively to a pure InAs and to a pure GaAs or AlAs substrate.

When $a_{\|}=a_{0}(\mathrm{InAs})$, both GaAs and AlAs are strained. The calculated VBO for GaAs/InAs, InAs/AlAs, and GaAs/AlAs is $0.27 \mathrm{eV}, 0.07 \mathrm{eV}$, and $0.34 \mathrm{eV}$ respectively, and the corresponding $\mathrm{CBO}$ is $0.32 \mathrm{eV},-1.10 \mathrm{eV}$, and $-0.78 \mathrm{eV}$.

When $a_{\|}=5.57 \AA \approx a_{0}(\mathrm{GaAs}) \approx a_{0}(\mathrm{AlAs})$, instead, only InAs shows a sizeable strain. The calculated VBO for GaAs/InAs, InAs/AlAs, and GaAs/AlAs is in this case $-0.27 \mathrm{eV}$, $0.83 \mathrm{eV}$, and $0.44 \mathrm{eV}$ respectively and the corresponding $\mathrm{CBO}$ is $0.71 \mathrm{eV},-0.95 \mathrm{eV}$, and $-0.36 \mathrm{eV}$ respectively. We notice that deviations from the transitivity rule up to $\approx 0.1 \mathrm{eV}$, definitely larger than our numerical accuracy, occur for this substrate, and can be ascribed 
to non neglegible local strain effects at InAs/GaAs(AlAs) interface.

Summarizing, the main result is that for all the three interfaces the type of alignment remains the same by changing $a_{\|}$from $a_{0}(\mathrm{InAs})$ (panel b)) to $a_{0}\left(\operatorname{In}_{0.75} \mathrm{Ga}(\mathrm{Al})_{0.25} \mathrm{As}\right)$ (panel c)). At variance, due to sizeale strain effects, when $a_{\|}=5.57 \AA \approx a_{0}(\mathrm{GaAs}) \approx a_{0}(\mathrm{AlAs})$ (panel d)) the band line-up for the GaAs/InAs interface changes from type II to type I, as already found in Refs. $12,44,45$.

\section{CONCLUSIONS}

We have estimated $\mathrm{VBO}=0.11 \mathrm{eV}$ for the $\mathrm{In}_{x} \mathrm{Ga}_{1-x} \mathrm{As} / \mathrm{In}_{y} \mathrm{Al}_{1-y}$ As heterojunction with homogeneous composition $x=y=0.75$, including spin-orbit and self-energy corrections to the calclauted LDA value. Neither different possible structural orders nor realistic accidental composition fluctuations around the nominal value can modify the VBO more than the estimated $\approx 0.1 \mathrm{eV}$.

Instead, we have predicted a maximum range of variability of about $1.1 \mathrm{eV}$ for the $\mathrm{VBO}$ at $\mathrm{In}_{x} \mathrm{Ga}_{1-x} \mathrm{As} / \mathrm{In}_{y} \mathrm{Al}_{1-y} \mathrm{As}$ heterostructures taking into account interfaces intentionally formed between different pure binaries and on different substrates. Neglecting self-energy corrections in this case - which could affect a little bit the individual band offset values but are not expected to change qualitatively its range of variability - the $\mathrm{VBO}$ goes from $0.83 \mathrm{eV}$ in the case of InAs/AlAs interface with a parallel lattice constant equal to the one of GaAs or AlAs, to $0.44 \mathrm{eV}$ in case of GaAs/AlAs unstrained interface, up to $0.07 \mathrm{eV}$ at InAs/AlAs on InAs substrate and $-0.27 \mathrm{eV}$ in the case of GaAs/InAs interface on GaAs substrate. The range of variation of the $\mathrm{CBO}$ is even larger, due to the larger effects of strain on the conduction band edges and energy gaps rather then on the valence band edges: the maximum range of variation is almost $1.8 \mathrm{eV}$, from $0.71 \mathrm{eV}$ occurring at GaAs/InAs interface with a parallel lattice constant equal to the one of GaAs, up to $-1.10 \mathrm{eV}$ at InAs/AlAs interface with a parallel lattice constant equal to the one of InAs.

\section{Acknowledgments}

We want to ackowledge L. Sorba for helpful discussions and a careful reading of the manuscript and A. Continenza for kind help when using the FLAPW code. Computational 
resources have been obtained partly within the "Iniziativa Trasversale di Calcolo Parallelo" of the Italian Istituto Nazionale per la Fisica della Materia (INFM) and partly within the agreement between the University of Trieste and the Consorzio Interuniversitario CINECA (Italy).

\section{APPENDIX A: STRAIN AND SPIN-ORBIT EFFECTS ON THE BAND EDGES}

\section{Valence band edges}

The strain shifts and splits the band edge manifolds of each constituent into the heavy hole $(\mathrm{HH})$, light hole $(\mathrm{LH})$ and split-off $(\mathrm{SO})$ states, which can be expressed as:

$$
E_{v, H H / L H / S O}^{S t r a i n}=E_{v, a v}^{0}+a_{v} \operatorname{tr} \epsilon+\Delta E_{v, H H / L H / S O}
$$

where $E_{v, a v}^{0}$ is the average of the valence band top edge manifold at $\Gamma$ in the unstrained compound, $a_{v}$ the valence band deformation potential taking into account the effect of the

hydrostatic component of the strain, and $\Delta E_{v}^{H H / L H / S O}$ account both for the effects of the shear strain and of spin-orbit coupling: ${ }^{25}$

$$
\begin{aligned}
\Delta E_{v, H H} & =\frac{1}{3} \Delta_{0}-\frac{1}{2} \delta E_{v, 001} \\
\Delta E_{v, L H} & =-\frac{1}{6} \Delta_{0}+\frac{1}{4} \delta E_{v, 001}+\frac{1}{2}\left[\Delta_{0}^{2}+\Delta_{0} \delta E_{v, 001}+\frac{9}{4}\left(\delta E_{v, 001}\right)^{2}\right]^{\frac{1}{2}} \\
\Delta E_{v, S O} & =-\frac{1}{6} \Delta_{0}+\frac{1}{4} \delta E_{v, 001}-\frac{1}{2}\left[\Delta_{0}^{2}+\Delta_{0} \delta E_{v, 001}+\frac{9}{4}\left(\delta E_{v, 001}\right)^{2}\right]^{\frac{1}{2}}
\end{aligned}
$$

where $\delta E_{v, 001}$ is the splitting due to the shear strain only, which be obtained directly from standard non relativistic band structure calculations of the constituent in the corresponding strain state. The final estimate of the valence band levels can be obtained from Eqs. A2 using the calculated $\delta E_{v, 001}$ and the experimental spin orbit splitting $\Delta_{0}$.

\section{Conduction band edges}

In order to study the effects of strain on the conduction band edges we have to distinguish two cases. The conduction band bottom for direct gap semiconductors like GaAs and InAs occurs at the $\Gamma$ point, it is non degenerate and its position with respect to the reference 
electrostatic potential depends only to the hydrostatic component of the strain through the conduction band deformation potential: ${ }^{25}$

$$
E_{c}^{\text {Strain }}(\Gamma)=E_{c}^{0}(\Gamma)+a_{c} t r(\epsilon)
$$

At variance, in case of AlAs the minimum of the conduction band occurs along the $<001>$ direction $\Delta$, close to the $\mathrm{X}$ point, it is six-fold degenerate in the unstrained semiconductor since there are six symmetry equivalent $<001>$ directions. Under strain, it is affected both by a shift due to the hydrostatic component of the strain through an indirect conduction band deformation potential $a_{c, \text { indirect }}$ and by a splitting due to shear components. In summary, and analogously to the expression of Eq. A1 for the valence band top edge manifold, we can write for the lowest state of the conduction band bottom:

$$
E_{c, \text { bottom }}^{\text {Strain }}(\Delta)=E_{c}^{0}(\Delta)+a_{c, \text { indirect }} \text { tr } \epsilon+\delta E_{c, \text { bottom }, 001}
$$

Similar expressions could be written for the other states of the conduction band manifold.

In order to give the best estimate for the conduction band offset, due to the limitation of the LDA, we can use the equations above without spin-orbit to extract from the nonrelativistic calculations of the materials in their strained state the quantities $\delta E_{v, 001}$ and $\delta E_{c, \text { bottom }, 001}$, and then we can use them again by inserting the experimental values of all the other band parameters $\left(E_{g}^{0}, a_{v}, a_{c}, a_{c, \text { indirect }}\right)$ for each material to get:

$$
\Delta E_{g}^{\text {strained }}=E_{g}^{0}+\left(a_{c}-a_{v}\right) \operatorname{tr} \epsilon-\Delta E_{v, H H / L H}
$$

where LH and HH holds respectively for GaAs and for InAs (their topmost valence states, see Table IV], and

$$
E_{g, \text { indirect }}^{\text {strained }}=E_{g, \text { indirect }}^{0}+\left(a_{c, \text { indirect }}-a_{v}\right) \operatorname{tr} \epsilon+\delta E_{c, 001, \text { bottom }}-\Delta E_{v, H H}
$$

for AlAs. Here $E_{g}^{0}=E_{c}^{0}-E_{v}^{0}+\frac{\Delta_{0}}{3}$ (experimental unstrained gap, without spin-orbit), and $\delta E_{c, 001, \text { bottom }}<0$ and $\Delta E_{v}^{H H / L H}>0$.

\section{APPENDIX B: ROLE OF $d$ ELECTRONS}

It has been shown ${ }^{46}$ that the $d$ electrons affect the properties of the II-VI zinc-blende compounds through $p-d$ repulsion and hybridization. In particular, they lead to a reduction 
of spin orbit splitting and increase of the valence band offset between common anion systems (by pushing up the Valence Band Maximum) with respect to calculations where $d$ electrons are considered as frozen (core) states.

In III-V systems, the $d$ electrons should play a minor role. In this Appendix we discuss in particular this point for our systems of interest by performing additional first-principle allelectron calculations on the bulk binary systems and their interfaces both considering $\mathrm{Ga}(\mathrm{In})$ $d$ electrons as core states or as relaxed (valence) states and we compare these results with the pseudopotential calculations. To this aim we use a full-potential linearized augmentedplane-wave code (FLAPW) $)^{47.48}$ using DFT-LDA, where spin-orbit splitting can be included as a perturbation on the semirelativistic calculation. This allows us to discuss the effect of $d$ electrons also on spin-orbit. We will consider the effects on the band structure of free-standing and strained GaAs and InAs bulks.

The calculated FLAPW equilibrium lattice constant $\left(\mathrm{a}_{e q}\right)$ for GaAs and InAs are 5.68(5.63) $\AA$ and 6.07(6.02) $\AA$ respectively when $d$ electrons are considered as core (valence) states. The slight increase of the lattice constant when $d$ electrons are frozen is along the trend of similar calculations..$_{.9 .50}$

The calculated spin-orbit splitting $\left(\Delta_{0}\right)$ does not change if $d$ electrons are treated as core or valence states, providing that the corresponding equilibrium theoretical lattice parameter is considered: it is equal to $0.36 \mathrm{eV}$ for GaAs and $0.39 \mathrm{eV}$ for InAs (the same as the experimental one). Therefore we can conclude that the influence of $d$ states on the spinorbit splitting of valence band maximum is negligible. This justifies our choice of using a posteriori the experimental values of the spin-orbit splittings to correct the non-relativistic LDA pseudopotential structure.

We have also studied the role of $d$ states when strain is applied, considering the same strain states used in our pseudopotential calculations both for GaAs strained over InAs $\left(a_{\|}=5.96\right.$ $\AA$, see Table IV) and for InAs strained over $\mathrm{Ga}_{0.5} \mathrm{Al}_{0.5} \mathrm{As}\left(a_{\|}=5.57 \AA\right.$, see Table IV). We choose to keep the same strain tensor in FLAPW and PWSCF calculations for a systematic comparison of the results, although the two different computational method would give slightly different equilibrium lattice constants for the binary compounds and consequently slightly different strain states. In these cases, we have used the elastic constants reported in Table [1 a slightly different value of $c_{i j}$ as calculated with the FLAPW method should lead to a negligible variation of the ratio $\frac{c_{11}}{c_{12}}$. The calculated $\delta E_{v, 001}^{G a A s}$ is equal to $-0.17(-0.14)$ 
$\mathrm{eV}$ and $\delta E_{v, 001}^{I n A s}=-0.35(-0.35) \mathrm{eV}$ for $d$ states treated as core (valence). Therefore, a small variation in the strain splitting effect is possible due to a different treatment of $d$ states, but very limited, here estimated within $0.03 \mathrm{eV}$.

* Electronic address: astroppa@ts.infn.it

$\dagger$ Electronic address: peressi@ts.infn.it

1 I. Vurgaftman, J.R. Meyer, and L.R. Ram-Mohan, J. Appl. Phys. 89, 5815 (2001).

2 M. Peressi, N. Binggeli, and A. Baldereschi, J. Phys. D 31, 1273 (1998).

3 A. Franciosi and C. G. Van de Walle, Surface Science Reports 25, 1 (1996).

4 F. Capasso and G. Margaritondo, Heterojunction Band Discontinuities: Physics and Device Application (Amsterdam: North-Holland, 1987).

5 H. Kroemer, Surf. Sci. 132, 543 (1983).

6 P. Offermans, P.M. Koenraad, J.H. Wolter, M. Beck, T. Aellen, and J. Faist, Appl. Phys. Lett. 83 (20), 4131 (2003).

7 G.J. de Raad, P.M. Koenraad, and J.H. Wolter, Surf. Sci. 556, 39 (2004).

8 Y. Dong, R.M. Feenstra, M.P. Semtsiv, and W.T. Masselink, Appl. Phys. Lett. 84, 227 (2004).

9 J. Shumway, A.J. Williamson, A. Zunger, A. Passaseo, M. De Giorgi, R. Cingolani, M. Catalano, and P. Crozier, Phys. Rev. B 64, 125302 (2001).

10 X. Zhou, Y. Gu, I.L. Kuskovsky, G.F. Neumark, L. Zheng, and M.C. Tamargo, J. Appl. Phys. 94, $7136(2003)$.

11 P. Roura, A. Vila, J. Bosh, M. Lopez, A. Cornet, J.R. Morante, and D.I. Westwood, J. Appl. Phys. 82, 1147 (1997).

12 T. Mattila, L.-W. Wang, and A. Zunger, Phys. Rev. B 59, 15270 (1999).

13 S. Froyen, A. Zunger, and A. Mascarenhas, Appl. Phys. Lett. 68, 2852 (1996).

14 Y. Zhang, A. Mascarenhas, and L.W. Wang, Appl. Phys. Lett. 80, 3111 (2002).

15 Y. Hsu, G. B. Stringfellow, C.E. Inglefield, M.C. DeLong, P.C. Taylor, J.H. Cho, and T.-Y. Seong, Appl. Phys. Lett. 73, 3905 (1998).

16 J. Shao, A. Dornen, R. Winterhoff, and F. Scholz, Phys. Rev. B 66, 035109 (2002)

17 F. Capotondi, G. Biasiol, I. Vobornik, L. Sorba, A. Cavallini, and B. Fraboni, J. Vac. Sci. Technol. B 22, 702 (2004). 
18 S.J. Chua, X.H. Zhang, S.J. Xu and X. Gu, J. Phys.: Condens. Matter 9, L279 (1997).

19 J.S. Nelson, A.F. Wright, and C.Y. Fong, Phys. Rev. B 43, 4908 (1991).

20 S.-G. Shen and X.-Q. Fan, J. Phys.: Condens. Matter 93151 (1997).

21 M. Peressi, S. Baroni, A. Baldereschi and R. Resta, Phys. Rev. B 41, 12106 (1990).

22 A numerical simulation using supercells and real atoms instead of the virtual crystal approximation has been done there only for $x=y=0.5$, considering an ordered (001) $1+1$ superlattice.

23 S. Baroni, A. Dal Corso, S. de Gironcoli, and P. Giannozzi, http://www.pwscf .org

24 O.H. Nielsen and R.M. Martin, Phys. Rev. B 32, 3792 (1985).

25 C.G. Van de Walle, Phys. Rev. B 39, 1871 (1989); C.G. Van de Walle and R.M. Martin, Phys. Rev. Lett. 62, 2028 (1989) and references therein.

26 M. Peressi and S. Baroni, Phys. Rev. B 49, 7490 (1994).

27 F.D. Murnaghan, Proc. Natl. Acad. Sci. USA 50, 697 (1944).

28 A. Baldereschi and M. Peressi, J. Phys.: Condens. Matter 5, B37 (1993).

29 S. de Gironcoli, P. Giannozzi, and S. Baroni, Phys. Rev. Lett. 66, 2116 (1991).

30 A. Zunger, S.H. Wei, L.G. Ferreira, and J.E. Bernard, Phys. Rev. Lett. 65, 353 (1990).

31 A.M. Saitta, S. de Gironcoli, and S. Baroni, Phys. Rev. Lett. 80, 4939 (1998); ibid., Appl. Phys. Lett. 75,2746 (1999).

32 J.C. Mikkelsen, Jr., and J.B. Boyce, Phys. Rev. Lett. 49, 1412 (1982); ibid., Phys. Rev. B 28, 7130 (1983).

33 In the $L$ and $F$ we have only In-Ga and In-In NNN pairs, and also Ga-Ga NNN pairs in the $S$ structure.

34 S.-H. Wei and A. Zunger, Phys. Rev. B 57, 8983 (1998).

35 A. Richter, M. Koch, T. Matsuyama, C. Heyn, and U. Merkt, Appl. Phys. Lett. 77, 3227 (2000).

36 S.I. Gozu, K. Tsuboki, M. Hayashi, C. Hong, and S. Yamada, J. Cryst. Growth 201, 749 (1999).

37 S.M. Wang, C. Karlsson, N. Rorsman, M. Bergh, E. Olsson, T.G. Andersson, and H. Zirath, J. Cryst. Growth 175/176, 1016 (1997).

38 S. Mendach, C.M. Hu, C. Heyn, S. Schnüll, H.P. Oepen, R. Anton, and W. Hansen, Physica E 13, $1204(2002)$.

39 J.L. Shieh, J.I. Chyi, R.M. Lin, and J.W. Pan, Electron. Lett. 30, 2172 (1994).

40 J.R. Waldrop, E.A. Kraut, C.W. Farley, and W. Grant, J. Appl.Phys. 69, 372 (1991).

41 The experimental spin-orbit energies are: $\Delta_{0}=0.34 \mathrm{eV}$ for GaAs, $0.28 \mathrm{eV}$ for AlAs and $0.39 \mathrm{eV}$ 
for InAs.

42 X. Zhu and S. G. Louie, Phys. Rev. B 43, 14142 (1991).

43 Basically, one can write $\Delta E_{g}^{q p}=\left(\epsilon_{c}^{L D A}+\delta \epsilon_{c}^{q p}\right)-\left(\epsilon_{v}^{L D A}+\delta \epsilon_{v}^{q p}\right)$, where $\delta \epsilon_{c, v}^{q p}$ is the quasi-particle correction to LDA eigenvalues. We derived $\delta \epsilon_{c, v}^{q p}$, from Ref. 42. In order to compare $\Delta E^{q p}$ with the experimental gap, one has to include a correction to the quasi-particle eigenvalues $\left(\delta r_{c, v}\right)$ and take into account the spin-orbit correction (which is not included in the previous formula). Finally, one has $\mathrm{R}=\left(\mathrm{E}_{g}^{e x p}+\frac{\Delta_{0}}{3}\right)-E_{g}^{L D A}=\delta \epsilon_{c}^{q p}-\delta \epsilon_{v}^{q p}+\left(\delta r_{c}-\delta r_{v}\right)$, where $\mathrm{E}_{g}^{e x p}$ and $\mathrm{E}_{g}^{L D A}$ are the experimental and LDA energy gap. Taking into account our estimate of $\mathrm{E}_{g}^{L D A}$, we rescaled $\delta \epsilon_{v}^{q p}$ according to $\frac{R_{2}}{R_{1}}$, where $\mathrm{R}_{1}$ is $\mathrm{R}$ quantity as derived from Ref. 42 and $\mathrm{R}_{2}$ as derived from our calculations.

44 K. Kim, P.R.C. Kent, A. Zunger, and C. B. Geller, Phys. Rev. B 43, 14142 (1991).

45 N. Tit, M. Peressi, and S. Baroni, Phys. Rev. B 48, 17607 (1993).

46 S.-H. Wei and A. Zunger, Phys. Rev. B 37, 8958 (1988).

47 H. J. F. Jansen and A. J. Freeman, Phys. Rev. B 30, 561 (1984).

48 The calculations for the binary compounds InAs and GaAs have been done with the wave function cutoff $K_{\max }=3.7$ a.u. Tests performed by increasing the cutoff up to 4.0 a.u. lead to a variation of relevant electronic properties less than of $0.01 \mathrm{eV}$. The wave function and the charge density (potential) expansion in the muffin-tin spheres include angular momenta up to $l=8$. The sphere radius for In, Ga and As are equal to 2.3, 2.3 and 2.2 a.u. respectively. The core charge spilling out of the spheres (when $d$ electrons are considered as core states) is treated using an exact overlapping charge method. The Brillouin zone sampling is the same of that used in pseudopotential calculations

49 B.I. Min, S. Massidda, and A.J. Freeman, Phys. Rev. B 38, 1970 (1988).

50 S. Massidda, A. Continenza, A.J. Freeman, T.M. de Pascale, F. Meloni, M. Serra, Phys. Rev. B 41, 12079 (1990). 
TABLE I: Relevant structural and electronic equilibrium parameters for bulk GaAs, AlAs, InAs. For AlAs we report the indirect/direct energy gap. Experimental data are reported in round brackets 1

\begin{tabular}{|c|c|c|c|}
\hline \hline & GaAs & AlAs & InAs \\
\hline$a_{0}(\AA)$ & $5.55(5.65)$ & $5.60(5.66)$ & $5.96(6.06)$ \\
$B(k B a r)$ & $760(784)$ & $750(773)$ & $670(579)$ \\
$c_{11}(k B a r)$ & $1240(1221)$ & $1130(1250)$ & $940(833)$ \\
$c_{12}(k B a r)$ & $520(566)$ & $560(534)$ & $540(453)$ \\
$E_{g}(e V)$ & $1.44(1.52)$ & $1.35(2.24) / 2.41(3.10)$ & $0.39(0.42)$ \\
\hline \hline
\end{tabular}


TABLE II: Calculated and experimental mismatches between binary semiconductors.

\begin{tabular}{|l|c|c|}
\hline \hline Mismatch (\%) & Theory & Experiment \\
\hline GaAs-AlAs & 1.0 & 0.1 \\
GaAs-InAs & 7.5 & 7.2 \\
AlAs-InAs & 6.4 & 7.1 \\
\hline \hline
\end{tabular}


TABLE III: Nearest-neighbour (NN) and next-nearest-neighbour distances (NNN) in $\mathrm{In}_{0.75} \mathrm{Ga}_{0.25}$ As and in $\mathrm{In}_{0.75} \mathrm{Al}_{0.25}$ As alloys in the three ordered structure considered in the text. We indicate with cat.-cat. all the possible pairs of cations. The error in the distances indicates their spreading in the structure considered. Units are $\AA$.

\begin{tabular}{|c|c|c|c|}
\hline \hline & $S$ & $L$ & $F$ \\
\hline \hline \multicolumn{4}{|c|}{$\mathrm{In}_{0.75} \mathrm{Ga}_{0.25} \mathrm{As}$} \\
\hline $\mathrm{NN}($ In-As $)$ & $2.57 \pm 0.01$ & 2.58 & $2.59 \pm 0.01$ \\
$\mathrm{NN}($ Ga-As $)$ & 2.45 & 2.43 & 2.41 \\
\hline $\mathrm{NNN}($ cat.-cat. $)$ & $4.15 \pm 0.07$ & 4.15 & 4.15 \\
$\mathrm{NNN}($ As-As $)$ & $4.07 \pm 0.16$ & $4.15 \pm 0.18$ & $4.146 \pm 0.22$ \\
\hline \hline \multicolumn{5}{|c|}{$\mathrm{In}_{0.75} \mathrm{Al}_{0.25} \mathrm{As}$} \\
\hline $\mathrm{NN}($ In-As $)$ & $2.58 \pm 0.01$ & 2.58 & $2.58 \pm 0.01$ \\
$\mathrm{NN}$ (Al-As) & 2.45 & 2.44 & 2.43 \\
\hline $\mathrm{NNN}$ (cat.-cat. $)$ & $4.15 \pm 0.07$ & 4.15 & 4.15 \\
$\mathrm{NNN}($ An-An) & $4.083 \pm 0.15$ & $4.15 \pm 0.16$ & $4.15 \pm 0.19$ \\
\hline \hline
\end{tabular}


TABLE IV: Calculated structural and band parameters for the three binaries GaAs, AlAs, InAs in three pseudomorphic structures: with a parallel lattice constant $a_{\|}=5.96 \AA$ (equal to that of bulk InAs), $a_{\|}=5.86 \AA$ (bulk $\operatorname{In}_{0.75} \mathrm{Ga}(\mathrm{Al})_{0.25} \mathrm{As}$ ), $a_{\|}=5.57 \AA$ (bulk GaAs or AlAs). For the energy gap, the values reported in this table are those for the minimum gap only (indirect in AlAs), and are obtained by taking from self-consistent calculations the effects of strain and by adding a posteriori an empirical correction from the comparison of experimental/theoretical data for the binary bulks.

\begin{tabular}{|c|c|c|c|}
\hline & GaAs & AlAs & InAs \\
\hline \multicolumn{4}{|c|}{$a_{\|}=5.96 \AA$} \\
\hline$\epsilon_{\|}$ & 0.075 & 0.064 & 0.000 \\
\hline$\epsilon_{\perp}$ & -0.064 & -0.063 & 0.000 \\
\hline$a_{\perp}(\AA)$ & 5.19 & 5.25 & 5.96 \\
\hline$\Delta E_{v, L H / H H / S O}(\mathrm{eV})$ & $0.44(\mathrm{LH})$ & $0.36(\mathrm{LH})$ & $0.13(\mathrm{HH}, \mathrm{LH})$ \\
\hline $\mathrm{E}_{g}(\mathrm{eV})$ & 0.47 & 1.59 & 0.42 \\
\hline \multicolumn{4}{|c|}{$a_{\|}=5.86 \AA$} \\
\hline$\epsilon_{\|}$ & 0.058 & 0.047 & -0.016 \\
\hline$\epsilon_{\perp}$ & -0.048 & -0.047 & 0.018 \\
\hline$a_{\perp}(\AA)$ & 5.28 & 5.34 & 6.07 \\
\hline$\Delta E_{v, L H / H H / S O}(\mathrm{eV})$ & $0.37(\mathrm{LH})$ & $0.29(\mathrm{LH})$ & $0.19(\mathrm{HH})$ \\
\hline $\mathrm{E}_{g}(\mathrm{eV})$ & 0.69 & 1.76 & 0.44 \\
\hline \multicolumn{4}{|c|}{$a_{\|}=5.57 \AA$} \\
\hline$\epsilon_{\|}$ & 0.005 & -0.005 & -0.065 \\
\hline$\epsilon_{\perp}$ & -0.004 & 0.005 & 0.075 \\
\hline$a_{\perp}(\AA)$ & 5.52 & 5.63 & 6.41 \\
\hline$\Delta E_{v, L H / H H / S O}(\mathrm{eV})$ & $0.13(\mathrm{LH})$ & $0.11(\mathrm{HH})$ & $0.41(\mathrm{HH})$ \\
\hline $\mathrm{E}_{g}(\mathrm{eV})$ & 1.45 & 2.25 & 0.47 \\
\hline
\end{tabular}


FIG. 1: Band structure of $\operatorname{In}_{0.75} \mathrm{Ga}_{0.25}$ As (top panels) and $\operatorname{In}_{0.75} \mathrm{Al}_{0.25}$ As (bottom panels) alloys in the (001)3+1 superlattice (S), luzonite (L) and famatinite (F) structures. Thick lines emphasize valence and conduction band edges. The zero of the energy scale is always set at the valence band maximum.

FIG. 2: Density of states of $\operatorname{In}_{0.75} \mathrm{Ga}_{0.25} \mathrm{As}$ and $\operatorname{In}_{0.75} \mathrm{Al}_{0.25}$ As alloys in the (001) $3+1$ superlattice (S), luzonite (L) and famatinite (F) structures. The zero of the energy scale is always set at the valence band maximum.

FIG. 3: Macroscopic average ${ }^{2}$ of the charge density $\rho_{\text {Macro }}$ (solid lines, right scale) and electrostatic potential $\mathrm{V}_{\text {Macro }}$ (dashed lines, left scale) for the $\operatorname{In}_{0.75} \mathrm{Ga}_{0.25} \mathrm{As} / \mathrm{In}_{0.75} \mathrm{Al}_{0.25}$ As heterojunction when the alloys are described using a luzonite structure (upper panel) and a (001) $3+1$ superlattice (lower panel). The position of the anionic planes is indicated in the $x$-axis.

FIG. 4: Calculated valence (conduction) band offsets for the (001) interfaces between the alloys (top panel a)) and binaries GaAs, AlAs and InAs pseudomorphically grown on a substrate with: b) $a_{\|}=5.96 \AA$ (equal to that of bulk InAs), c) $a_{\|}=5.86 \AA$ (bulk $\left.\left.\operatorname{In}_{0.75} \mathrm{Ga}(\mathrm{Al})_{0.25} \mathrm{As}\right), \mathrm{d}\right) a_{\|}=5.57 \AA$ (bulk GaAs or AlAs). The band alignments are calculated between $E_{v}$ and $E_{c}$, the highest valence (lowest conduction) states including spin-orbit effects. We indicate in boldface the unstrained compounds. Values reported here include spin-orbit but not self-energy corrections. 

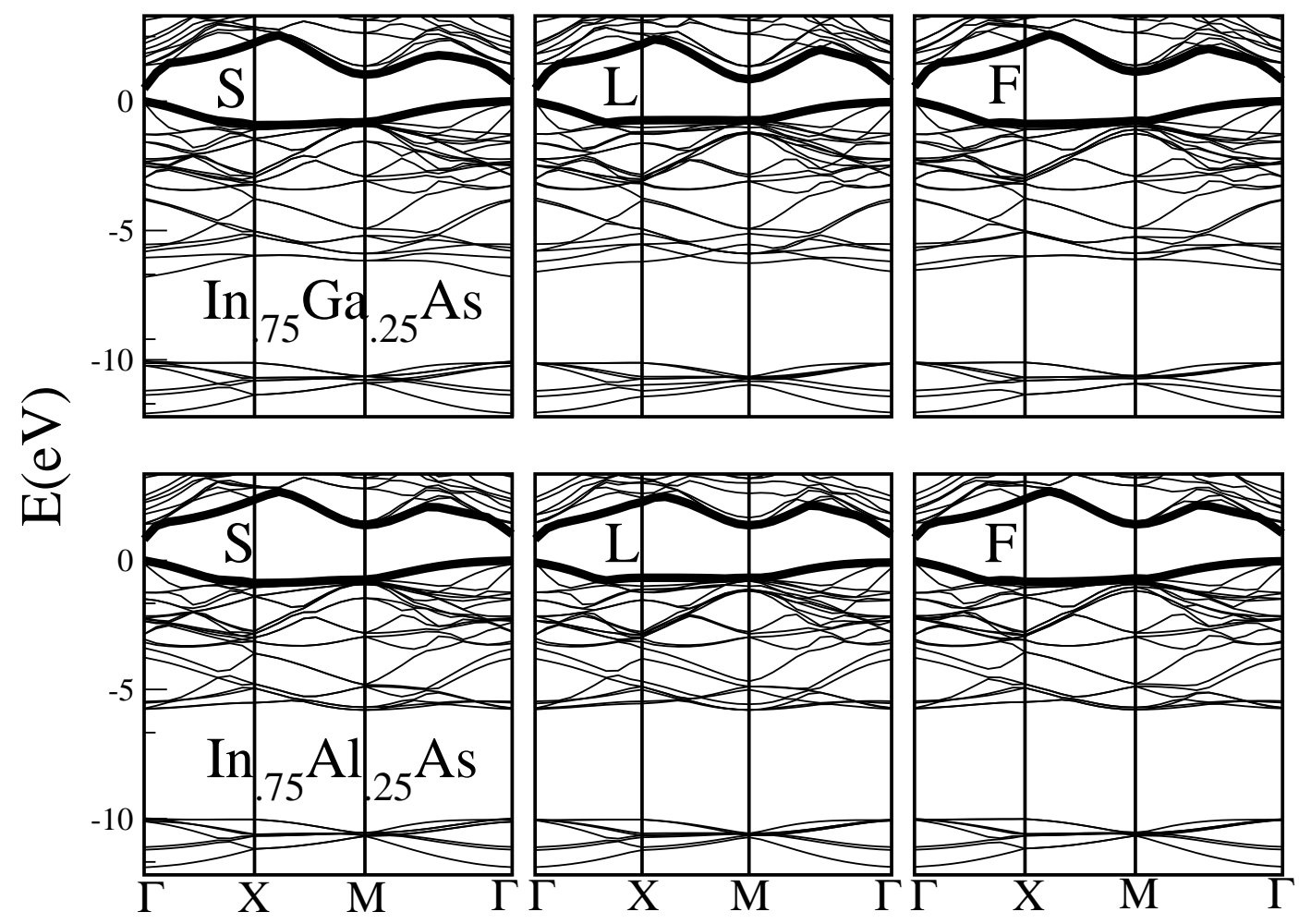

Fig. 1 


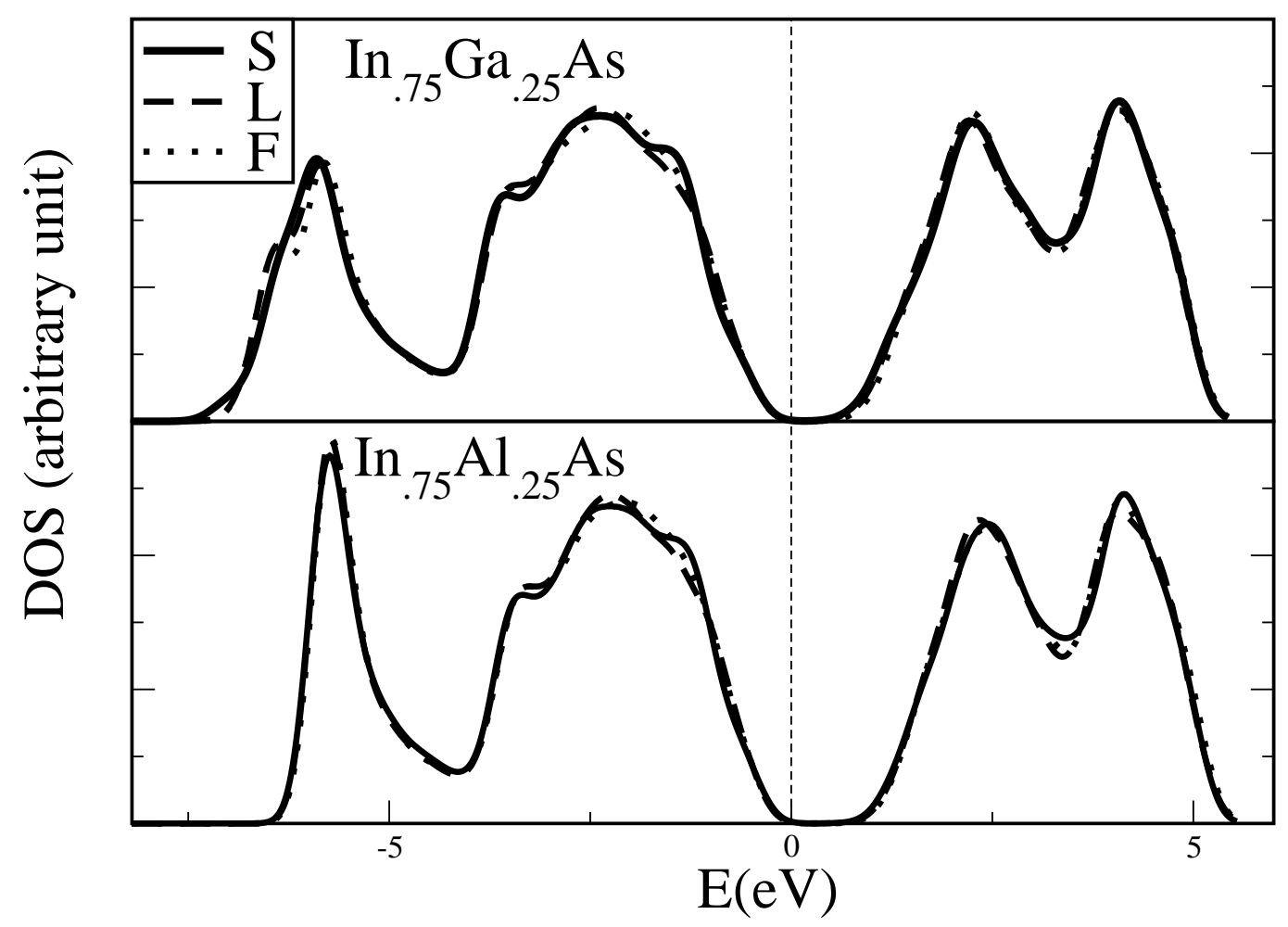

Fig. 2 


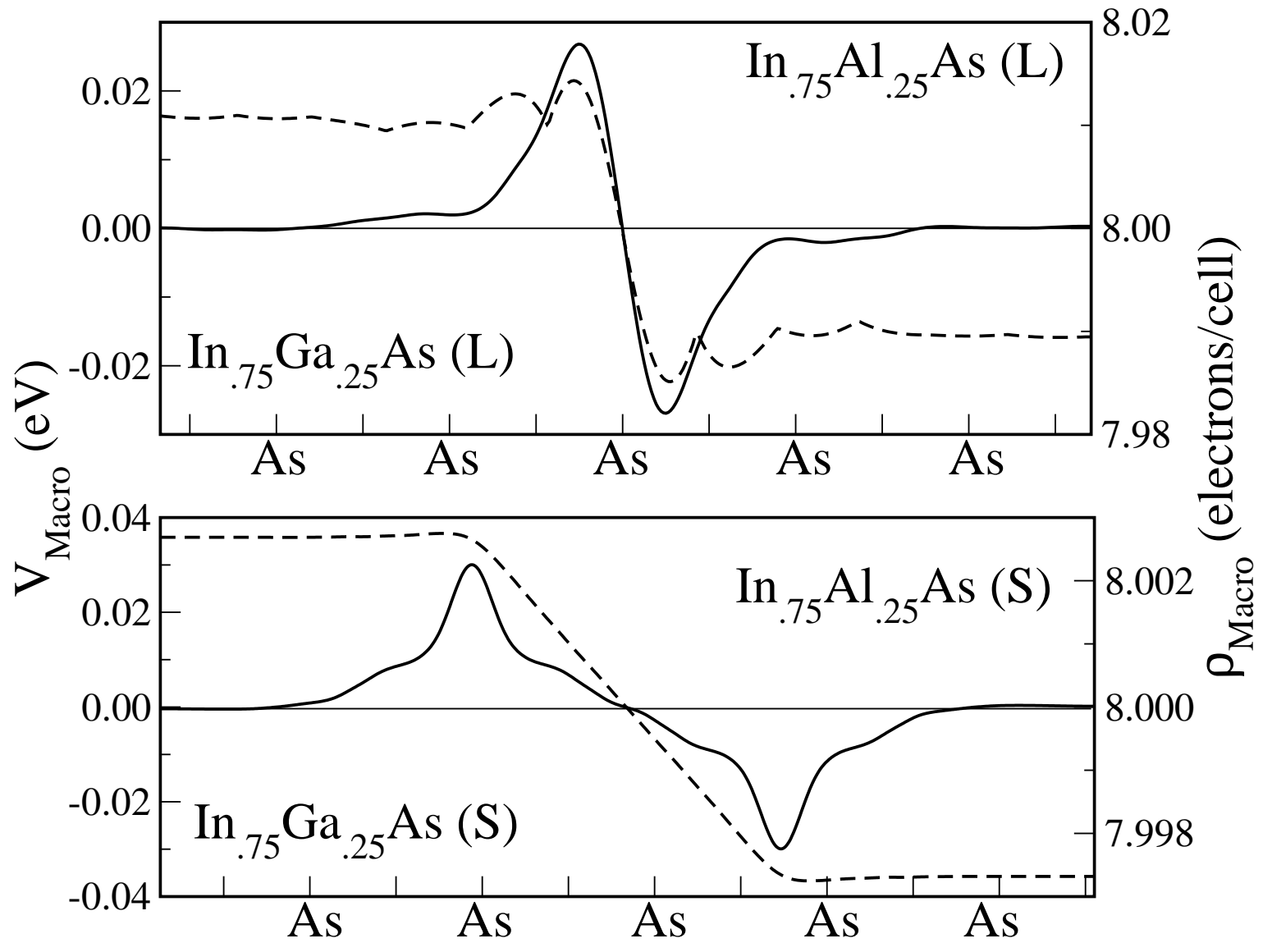

Fig. 3 
a)

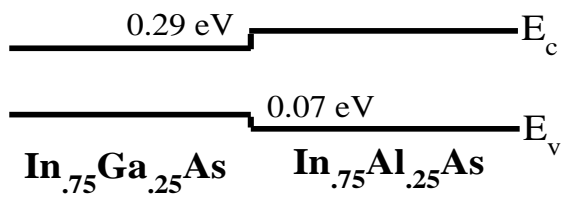

b) $0.32 \mathrm{eV}$

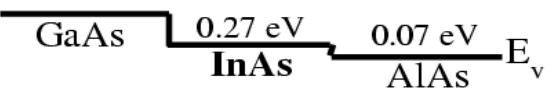

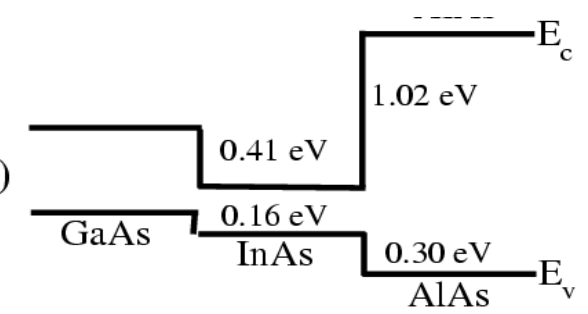

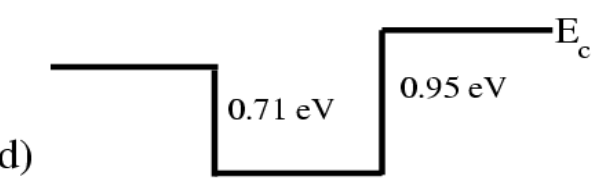

\begin{tabular}{ll}
$0.27 \mathrm{eV}$ & InAs \\
\hline GaAs & $\begin{array}{c}0.83 \mathrm{eV} \\
\text { AlAs }\end{array} \mathrm{E}_{\mathrm{v}}$
\end{tabular}

Fig. 4 